Le portiQue Lortique

Revue de philosophie et de sciences humaines

$5 \mid 2000$

Passages du siècle

\title{
Entretien avec Jean-Toussaint Desanti
}

$\mathrm{X}^{\mathrm{e}}$ Nuit culturelle28 mars 1998

\section{Nicolas Antenat}

\section{CpenEdition}

Journals

Édition électronique

URL : http://journals.openedition.org/leportique/412

DOI : $10.4000 /$ leportique.412

ISSN : $1777-5280$

Éditeur

Association "Les Amis du Portique"

Édition imprimée

Date de publication : 1 janvier 2000

ISSN : 1283-8594

Référence électronique

Nicolas Antenat, «Entretien avec Jean-Toussaint Desanti », Le Portique [En ligne], 5 | 2000, mis en ligne le 24 mars 2005, consulté le 26 mars 2021. URL : http://journals.openedition.org/leportique/412 ;

DOI : https://doi.org/10.4000/leportique.412

Ce document a été généré automatiquement le 26 mars 2021.

Tous droits réservés 


\title{
Entretien avec Jean-Toussaint Desanti
}

Xe Nuit culturelle28 mars 1998

\author{
Nicolas Antenat
}

1 À l'occasion de la $X^{e}$ Nuit Culturelle, manifestation nancéenne regroupant conférences, lectures et expositions artistiques diverses, nous avons eu le plaisir d'entendre le philosophe JeanToussaint Desanti nous parler, à partir d'un extrait du film Les Temps modernes de Charlie Chaplin, de la constitution de ce que Sartre nommait "un groupe en fusion » et d'une manière plus fondamentale de la construction et de l'effondrement des systèmes de croyances. Pour connaitre la teneur de ce propos, nous renvoyons le lecteur au livre de J.-T. Desanti, Un destin philosophique ou les pièges de la croyance ${ }^{1}$, et préférons ici retranscrire l'entretien qui suivit et qui fut, dans l'intimité entre un public hétéroclite et ce philosophe à la parole chaude et colorée, un rare et précieux moment de philosophie. C'est, en effet, par cette parole sérieuse et malicieuse qu'il nous permet de faire l'expérience de ce que Platon nommait la sunousia, la fréquentation vivante d'un maitre véritable. Car, au-delà des questions précises qu'il aborde en phénoménologue, mathématicien et historien de la philosophie, c'est quelque chose du statut de la philosophie contemporaine et de la situation du philosophe lui-même que l'horizon de sa pensée pénétrante dessine.

2 - Public : Finalement nous avons fait, ce soir, l'expérience de ce que pouvait être un groupe en fusion, avec sa fragilité, la menace de déconstitution qui l'anime, et je crois que ce qui était important dans ce qui a été dit ce soir c'est qu'un groupe en fusion n'est pas quelque chose de solide. Ce n'est pas quelque chose d'organique même si nous sommes à peu près assurés d'avoir des corps biologiques. Les groupes dont vous nous avez parlé ne sont pas solides. À partir de là je crois que nous pouvons être amenés à différencier un symbole d'un simple signal qui, lui, entretient des rapports relativement solides avec ce qu'il appelle. Est-ce qu'on pourrait, toujours en nous concentrant sur notre exemple du drapeau, dire que celui-ci est passé d'un certain état malgré tout proche du signal - il signale le danger - à un état de symbole. Pouvez-vous clarifier ce passage pour nous? 
- Jean-Toussaint Desanti : Oui, mais là il faut faire la distinction entre la manière dont je l'ai présenté dans Un destin philosophique et la façon de s'en servir dans le film. Ce n'est pas la même chose, le même phénomène. Dans le film il y a déjà une manifestation, les ouvriers se meuvent derrière des pancartes et c'est ce pauvre Charlot qui se retrouve coincé. En prenant ce bout d'étoffe il se désigne comme le meneur. J'ai en effet modifié l'exemple, je l'ai modifié pour les besoins de ma démonstration. Je n'ai retenu que le drapeau. Les ouvriers, les gens qui participent à la manifestation, sont simplement là. Ils sont disséminés, certains sont assis, d'autres discutent de la situation de revendication. Mais c'est le drapeau, la vue du drapeau, qui coagule tout cela et ils passent, pour ainsi dire, du fait d'être simplement là à discuter de certains problèmes, à la fusion.

- Qu'est-ce qui, dans cette fusion, donne tout d'un coup l'apparence d'une certaine solidité ?

5 - C'est l'opposition, dans cette partie du moment où les flics arrivent (dans mon exemple, pas dans le film), qui fait la solidité du groupe, la fusion contre la menace, contre la menace de la violence de l'État, contre la violence policière. À ce moment-là le groupe est sur le chemin, sinon d'une institutionnalisation, au moins d'une organisation provisoire. Si les flics commencent à taper dessus il va falloir savoir comment répondre: est-ce qu'on va fuir ou au contraire leur tomber dessus. À ce moment-là il y a une récupération sur un agir commun, sur un projet commun et alors le groupe se renforce ; le groupe en fusion se renforce. Et si d'autres groupes en fusion se constituent à ce moment-là - comme en mai 1968 tous ces groupes en fusion qui se sont constitués autour des barricades avec des gens qui parfois ne se connaissaient pas -, si tout ceci se répond et est en devenir, s'ils se font signe, s'ils s'envoient des messages pour se dire voilà ce qui se passe ici ou là, c'est alors une configuration plus importante que le groupe en fusion qui est en train de se former; c'est une configuration, sinon de parti, au moins de projet provisoire commun : celui d'essayer de ne pas se faire massacrer par la police.

6 - Est-ce que cela voudrait dire que l'on ne peut s'identifier que par rapport à un adversaire?

7 - Forcément, cela aide !... On ne peut s'identifier que par rapport à ce qui est devant et dans la mesure où l'on identifie ce qui est devant, ce qui s'oppose. Moi, en ce moment, je m'identifie comme quelqu'un qui fume la pipe.

8 - Mais ceux qui ne la fument pas ne sont pas pour autant des adversaires par rapport auxquels vous pouvez vous identifier!

9 - Non, ce n'est pas celui qui ne fume pas qui est mon adversaire. C'est le tabac mon adversaire, je consomme mon adversaire ! Je m'identifie à ce moment-là.

10 - Est-ce que l'identification peut faire le consensus ?

11 - À travers le consensus il n'y a pas d'identification, il n'y a que l'effacement mou de chacun ; l'effacement mou de chacun dans l'abstraction des rapports de marché.

12 - Cela peut conduire à l'apologie de la haine.

13 - Un tout petit peu de haine n'est pas mauvais à condition qu'elle ne se déploie pas en système, qu'elle n'envahisse pas la personnalité tout entière, la société tout entière.

14 - C'est toute la problématique du fascisme. 

philosophique, une positivité de l'idée de fascisme?

19 philosophiquement, ce qu'il peut y avoir de positif dans le fascisme. Le fascisme stalinien, à la racine, se présente comme une réponse à l'état de l'après guerre...

- Le fascisme implique également la fascination et ne serions-nous pas aujourd'hui dans une époque de fascisme matérialiste?

- Nous sommes à une époque de fascisme larvé. Il y a un grand nombre de figures du fascisme aujourd'hui encore.

- Peut-être serait-il plus opportun de revenir à la question de la croyance et du malentendu. Paradoxalement vous avez laissé entendre précédemment que, d'un certain point de vue, le malentendu est premier. Comment sortir de ce paradoxe ? Est-ce en disant que la condition de possibilité de la communication c'est que le malentendu soit possible, sans dire qu'il doit nécessairement avoir lieu, ou est-ce que cette manière de sortir du paradoxe, en choquant le bon sens, ne vous satisfait pas?

23 - Là où il n'y a pas la menace du malentendu, là où il n'y pas la manifestation d'une distance de la parole de chacun à la parole de l'autre, il peut encore y avoir un phénomène de projection de sens, parce que chacun reste dans son immobilité. C'est la mobilité du gestuel signifiant qui, portant le risque local du malentendu, constitue en même temps le champ dans lequel le malentendu se dépasse. C'est le propre de la parole. Lorsque vous êtes agressés dans la rue, ou vous fuyez ou vous parlez. Mais si vous commencez à parler c'est un autre rapport qui s'institue dans le premier ; l'autre ne peut pas rester tout à fait muet et s'il commence à parler alors il est perdu, il renoncera à l'agression.

- Vous avez, auparavant, parlé du sens essentiellement comme d'un recouvrement, recouvrement d'un écart primordial et d'un écart fondamental. Le sens serait-il dû uniquement à la parole, serait-il une invention, une création ? Les choses ne font-elles pas sens par elles-mêmes?

- Les choses se font signe, elles exigent du sens dans la mesure où elles sont simplement un écart. Elles sont en écart les unes relativement aux autres de quatre façons : - elles sont d'abord un écart en ceci que chacune a son mode de surgissement propre, sa provenance, mais qui n'est jamais là. C'est son horizon indéterminé. Être là, ceci ne m'apprend rien sur l'identification de la chose qui est là. C'est le premier horizon de présentation, l'horizon de provenance qui comporte aussi l'indication d'un écart temporel indéterminé qui reste toujours implicite. - Puis il y a la façon dont la chose qui est là se trouve mise en relation, saisie, donnée en relation avec l'autre dans un horizon de coexistence. Cette relation de coexistence n'est pas seulement le contenant des choses, ce n'est pas seulement un espace que je pourrais vider et qui, étant vidé, serait ce sur quoi les choses reposent. Cet espace ne se perçoit pas, je perçois une spatialisation, la coexistence dans l'écart. C'est le deuxième horizon de 
présentation de la chose - Il y en a un troisième qui est que toute chose exige d'être désignée relativement aux autres, exige d'être saisie en tant que coexistante relativement aux autres dans un même champ, qui est le champ de la spatialité du monde. Ceci diffère de la manière dont chaque chose renvoie à une autre, comme devant se désigner l'une relativement à l'autre. C'est un autre horizon de désignation possible, la façon dont, par exemple, le briquet renvoie à la pipe. À ce moment-là mon corps singulier, particulier, mon corps vivant - non pas mon corps objectif qu'étudient les médecins et les biologistes, mais le corps que je vis, avec sa spatialité propre, interne, la différence entre mes deux oreilles, mon nez, mes yeux... que je saisis immédiatement - est toujours intéressé, partie prenante dans cette façon dont les objets se répondent les uns aux autres et, pour ainsi dire, se parlent les uns aux autres et nous parlent. Cela, c'est le troisième horizon.- Le quatrième qui rassemble tout, dans lequel tout se rassemble, c'est l'horizon du symbolique. Horizon dans lequel les désignations peuvent être gestuelles, verbales, articulées, dans lequel le champ de désignation se constitue, pour ainsi dire, pour lui-même dans une forme de relation qui lui est propre et qui est, en gros, le champ de la langue tant que celle-ci est parlée, tant qu'elle est opérée.

C'est l'unité de ces quatre horizons qui fait notre rapport au monde.

- Mais où et à quel moment se fait cette unité ?

- Dans la quatrième !... C'est-à-dire dans le rapport à autrui.

- Je voudrais savoir quel sens vous donnez au mot "parole" quand vous dites : "les choses parlent ". Vous évoquiez auparavant le mot sens... Qu'est-ce que " le sens du monde" ? Et qu'est-ce que c'est que cette exigence des choses d'être dites? Toute la philosophie analytique ramène cela à de la pure métaphore.

- Je le sais bien. Les choses ne parlent pas, elles nous parlent, elles nous concernent.

- L'expérience muette à laquelle Merleau-Ponty a fait un tel sort, c'est l'expérience humaine, mais qu'est-ce que le sens des choses s'il n'y pas quelqu'un pour le dire?

- L'expérience muette !... c'est le mot «muet » qui me gêne. Finalement c'est très orgueilleux de la part du philosophe, l'expérience reste muette tant que lui n'a pas parlé. Elle ne l'est pourtant pas, elle est première, elle est toujours déjà parlée par l'autre, toujours déjà présentée dans ce jeu du rapport symbolique qui constitue le signifiant de tout mode de présentation de ce qui se déploie comme appartenant à un monde localement déterminé, localement représenté. C'est en se sens que je dis que les choses nous parlent, dans la mesure où elles exigent la reprise dans la parole commune, dans le partage, dans l'échange qui est gestuel, dans la mobilité réciproque et signifiante de nos corps les uns pour les autres. Le premier signifiant c'est le corps de l'autre, pour chaque homme.

- C'est ce qui fait que les choses sont dicibles mais pas disantes !

- Elles ne disent pas, les choses ne disent rien. Ou bien elles ne se manifestent pas du tout, ou bien elles ne se déploient dans aucun champ de présence et alors, en effet, il n'y a pas d'expérience. Ou bien elles se manifestent comme définies, identifiables ou non, mais en tous cas exigeant la reprise dans un champ de connaissance ou d'expérience et alors elles exigent la parole pour subsister dans leur statut de choses manifestes. C'est pourquoi on les marque, même si la marque est purement interne, on les marque dans la mesure où on se les remémore. On se meut dans ce champ de la 
mémoire, de la remémoration. Lorsque je dis « cet arbre ", c'est toujours un autre, son identité ne se saisit que dans ce mouvement de remémoration de ses manifestations, ce paraître temporellement situé et donc toujours marqué. Un présent qui n'est pas marqué disparaît.

- Ce soir vous avez beaucoup parlé de la parole justement, la parole comme pont signifiant traversant un écart fondamental entre les choses. Mais la parole ne creuse-t-elle pas au contraire cet écart puisque nous essayons toujours de nous mettre d'accord sur le sens, puisque nous polémiquons sans cesse sur le sens des mots et des choses?

- Bien entendu, la parole recouvre sans combler jamais. La parole est toujours menacée, elle est toujours menacée d'effondrement. théorème mathématique sur le même mode que celui sur lequel se constitue ma croyance en la réalité de la chose présente dans ma perception. Mais une chose est de distinguer un mode de croyance, autre chose est de distinguer les formes d'organisation des systèmes de croyance qu'on finit par habiter. C'est ce qui arrive par exemple au mathématicien lorsqu'il croit qu'il y a des objets mathématiques situés en monde et qu'il doit organiser. Cela, c'est la constitution d'un système de croyance. Évidemment c'est une illusion. Il n'y a pas de monde mathématique dans lequel on habite. D'une certaine façon le mathématicien habite ces champs théoriques localement, mais il ne les surmonte pas, il ne les survole pas. Nous ne pouvons 
surmonter, survoler, nous ne pouvons prendre une vision enveloppante, globale, relativement à aucune des constellations de sens que nous dévoile notre expérience seconde des formes du temps, des formes spatio-temporelles. Tout geste de désignation est assigné localement et il n'y a pas de sujet qui puisse survoler tout cela. Ce serait être dans l'illusion pure et simple, dans le semblant.

- C'est un autre type d'univers de croyance. Mais est-ce que, précisément, l'analyse des phénomènes de croyance permettrait une prise de distance à l'égard de ces phénomènes?

- Je dis et pense qu'on ne peut pas. On ne peut pas échapper au caractère prégnant de « l'être-là » du monde. Il y a un parti pris pour le monde, qui est constitutif du monde.

- N'est-ce pas ici la question du sens critique qui se pose ? Est-ce que le fait de réfléchir, comme vous l'avez fait, sur le mode de constitution d'une croyance confère plus de sens critique?

50 - Plus de défiance! Plus de défiance à l'égard des engagements non-réfléchis.

51 - N'est-ce pas seulement le privilège de quelques-uns ?

52 - Chacun y connaît toujours quelque chose! Il ne faut pas se méprendre. Nul n'est ignorant !... Pourvu qu'il sache parler.

- Ici, automatiquement, c'est la question du pouvoir qui arrive.

- En effet, mais la question du pouvoir c'est encore une autre affaire. Aucun pouvoir ne peut se déployer ni apparaître s'il ne dispose pas d'un système de croyance renouvelable et sans cesse réassumé, s'il ne dispose pas de ces modalités d'institution de la fidélité aux croyances. C'est la servitude volontaire!

55 - Autre chose. Pouvez-vous nous en dire un peu plus sur votre relation à Stendhal. Stendhal est un homme de convictions. Vous nous avez expliqué comment vous avez changé de convictions, de croyances. Comment s'est effectué ce changement et que signifie-t-il pour vous?

56 - C'est tout le problème. Comment un système de croyance prégnant et apparemment solide se défait. Pour nous c'est simple; enfin, cela n'a pas été simple à vivre, c'est le phénomène qui est simple. À un moment nous avons été saisi par un phénomène historique qui a été le vingtième congrès du parti communiste de l'Union Soviétique dans lequel nous avons été pris sans le savoir - d'autres savaient, mais nous nous ne voulions pas le savoir pris que nous étions dans le système de renforcement de notre mode de capture dans le champ du parti communiste français. À un moment donné ce système de capture commence à se fissurer en un point, la fissure peut être tout à fait locale, elle peut être recouverte, on peut encore trouver des moyens pour peu que le système fondamental de croyance subsiste, des moyens théoriques, des motivations théoriques, pour recouvrir la fissure. On peut aussi se donner des motivations historiques, on peut se dire que c'est parce que l'Union Soviétique était ceci ou cela, etc., mais on retombe toujours dans le même système fondamental de croyance et on finit presque en fondateur du parti communiste. Et puis il peut arriver, et c'est ce qui arrive le plus souvent, que tout le système des modalités de renforcement de tout le jeu (de la force des images) qui fait que la croyance se constitue comme quelque chose d'apparemment solide, ne s'effectue plus. Qu'est-ce que cela veut dire? Cela veut dire que la parole symbolique, le poids de la parole symbolique issue du parti disparait. Le poids ne s'exerce plus, cette parole n'est plus entendue, elle devient morte. À ce 
moment-là il n'y a plus de convictions, ce n'est pas que l'on perde le sens du rapport au monde, que l'on sombre dans l'incohérence et la folie, mais un autre mode de cohérence, à la fois dans sa vie et dans ses rapports sociaux avec les autres, est exigé. Voilà, on change, on n'effectue plus le rapport au monde de la même façon. Je vous donne un exemple quand même extraordinaire : dans ma jeunesse j'étais trotskiste, j'ai appris les procès en Union Soviétique en 1935-1936, jusqu'en 1937, et je me suis dit "c'est abominable, ces salopards sont en train de détruire toute l'élite du parti bolchevique » et j'étais contre les procès de Moscou. Là, arrive la guerre, arrive l'attaque de l'Union Soviétique par les Allemands, arrive la retraite de l'armée soviétique. Tout en étant trotskiste je commençais à être très inquiet, et à ce momentlà, chose très étrange, l'attitude toute négative, l'attitude de refus que j'avais eu à l'égard des procès de Moscou s'est absolument effacée comme si le passé changeait de forme, exactement comme dans ces figures où l'on distingue la forme et le fond - les gestaltistes ont développé tout cela -, c'est la forme d'organisation de la relation présent passé, le passé étant ce fond, qui a changé. Et ce qui a informé le passé c'était ce qui se passait à Stalingrad! Là se constitue un bouleversement des modes de croyance et une installation dans un autre système de croyance en devenir. C'est comme cela que l'on adhère à un parti, c'est comme cela que ça s'est produit en 1933, par exemple, lorsque le système fonctionnait avec sa circularité propre, la parole du dirigeant se répercutant sur l'activité quotidienne du parti de manière doctrinaire. On dit toujours que le parti communiste est un parti comme les autres mais ce n'est justement pas un parti comme les autres. Dans notre esprit c'était un parti qui avait, pour ainsi dire, une fonction ontologique.

- Vous avez dit ce soir à plusieurs reprises qu'il n'y avait pas d'au-delà de la croyance, qu'il n'y avait pas de métalangage mais vous distinguez tout de même deux parties dans votre vie : une partie où vous avez cru et une partie où vous analysez ce que c'était que d'avoir cru; il a y donc bien deux rapports à la croyance...

-...à un contenu de croyance, mais pas au phénomène de la croyance. Je continue à croire à ce que je fais, mais je ne crois plus ce que j'ai cru. Je crois à ce que je fais sinon je ne pourrais plus rien faire. Le phénomène de la croyance est, pour employer le langage banal des philosophes, récurrent en tant que phénomène vide, indéterminé; on ne peut pas ne pas croire, on ne peut pas ne pas chercher à savoir.

- Il n'y aurait donc qu'une relativité entre différents modes de croyance ?

- Pas des modes, car les modes de croyance sont enracinés profondément dans des systèmes de croyance. Les contenus de croyance, eux, sont toujours relatifs pour moi.

61 - Est-ce que la constitution d'un univers de croyance ne suppose pas un rapport spécifique de la parole au malentendu ? En ce sens où, comme nous l'avons vu dans le film de Charlot, ce qui crée le malentendu c'est qu'il ne s'explique pas, que la parole est coupée ; c'est une fonction du malentendu qui est différente de celle dont nous avons parlé précédemment, qui, elle, est dynamique et pousse les personnes à s'expliquer les unes avec les autres, qui ne met jamais de termes au dialogue. Est-ce que la constitution d'un univers de croyance n'est pas liée à la fin du dialogue et à un rôle du malentendu dans la parole très différent de celui présent dans tout dialogue? 
62 - C'est plus que du malentendu dans ce cas-là, c'est une véritable cassure, une discontinuité dans le jeu des rapports symboliques. C'est à ce moment-là que le système de croyance exige le renforcement de la chaîne symbolique ; si ce renforcement ne se produit pas alors c'est le monde de ce genre de croyance, de cette espèce de croyance, qui s'effondre. Le fait que Charlot ait levé ce drapeau par hasard, de façon, pour ainsi dire muette, et qu'il se soit en définitive trouvé berné puisque c'est lui que l'on emmène au poste comme le meneur qu'il n'est pas, apparaît justement comme cette cassure, cette discontinuité. Alors que va-t-il faire? C'est son affaire, c'est à lui de s'en préoccuper. Un moment vient où le sort d'un système de croyance peut paraître dépendre de l'action hasardeuse d'un seul individu.

\section{- Ce que vous dites là est-il optimiste ou pessimiste ?}

- Je crois que je ne me fais pas trop d'illusions là-dessus, je ne crois pas que l'on peut raccommoder facilement une telle discontinuité, à ce point radicale. C'est très dangereux.

- Pouvons-nous aller plus loin ; vous avez parlé de système de croyance, de mode de croyance, vous avez insisté sur le fait qu'il nous est impossible de nous situer hors de la croyance. Pour replacer la question dans un domaine qui vous est familier, celui des mathématiques, la coexistence de plusieurs géométries - celle d'Euclide bien évidement, celles de Lobatchevski et de Riemann également - pose le problème de la validité...

- Absolument, dans la mesure où ils sont cohérents et où on peut les représenter les uns dans les autres!

- Là, vous vous intéressez à la question de la validité, mais que faites-vous de la question de la vérité, puisque nous parlons ici de la croyance. Certes, c'est une grande question, qui est certainement plus intéressante que nécessaire, mais peut-on privilégier uniquement la validité, préférer un système mathématique plutôt qu'un autre, être trotskiste et ne plus l'être, en fonction des conditions de réalité ? Derrière cela que reste-t-il, qu'en est-il de la question de la vérité et de sa recherche?

- Diable... la question de la vérité !... Je vais vous raconter une histoire qui n'a rien à voir : celle d'Aristote. Dans le livre II de la Physique, vers la fin, Aristote cherche à énoncer les principes qui peuvent servir de fondement à une science du mouvement. À un moment donné il dit : " les anciens ont mis les contraires au nombre des principes » et il ajoute - je vous le dis en grec car c'est très intéressant -

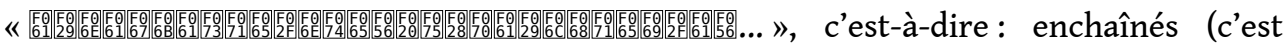
très fort) par ce qui était manifeste (non pas par la vérité) en l'absence de justification. C'était la situation fondamentale de l'être-là. C'est quand nous sommes enchainés au manifeste que la question de la vérité se pose. On ne défera jamais cette chaîne, c'est-àdire le lien qui nous rassemble au manifeste, jamais. Seulement, du fait que ce manifeste est lui-même une structure significative et qu'il est à la fois en écart relativement à lui-même et en écart relativement à la façon dont nous pensons avoir affaire à lui, de ce fait le problème de la vérité se transforme. Il subit, quasiment au sens mathématique du mot, une transformation. Il change de forme, les relations qui le constituent changent de forme et de statut. Elles deviennent des relations de validité, des relations d'adéquation de l'énoncé à l'état de chose. Mais à l'horizon reste toujours la chaîne, forcés que nous sommes par le manifeste. C'est la structure propre du 
manifeste qui pose la question de la validité des énoncés, des énonciations et de leurs formes. C'est pourquoi les problèmes de validité sont infinis et indéfinis, ils sont l'expression de cet enchaînement primordial au manifeste. Être enchaîné au manifeste cela veut dire également le suivre dans ses contours, dans ses mises à distance, le ressaisir soit dans la description soit dans la théorie, peu importe, car seul le développement est différent... C'est pourquoi je ne distinguerai pas recherche de

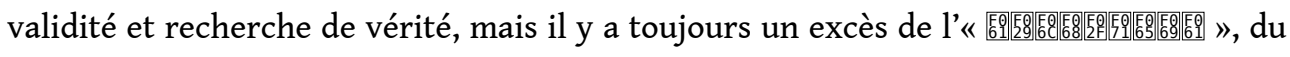
manifeste sur les procédures de validité.

Réalisé et transcrit par Nicolas Antenat.

\section{NOTES}

1.. Éditions Grasset, Biblio Essais, 1982. Pour l'utilisation du film de Chaplin, se reporter à la page 61 : «Charlot, s'étant par hasard emparé du drapeau rouge qui sert à signaler des travaux sur la voie publique, se trouve, de ce fait, avoir pris la tête d'une manifestation ». 